\title{
À la racine... ou l'histoire souterraine de la venue du Théâtre du Soleil à Lausanne. Portraits croisés d'Omar Porras et d'Ariane Mnouchkine
}

\section{Brigitte Prost}

\section{CpenEdition}

Journals

Édition électronique

URL : https://journals.openedition.org/edl/3246

DOI : $10.4000 /$ edl.3246

ISSN : 2296-5084

Éditeur

Université de Lausanne

\section{Édition imprimée}

Date de publication : 24 septembre 2020

Pagination : 285-300

ISBN : 978-2-940331-74-1

ISSN : 0014-2026

\section{Référence électronique}

Brigitte Prost, « À la racine... ou l'histoire souterraine de la venue du Théâtre du Soleil à Lausanne. Portraits croisés d'Omar Porras et d'Ariane Mnouchkine », Études de lettres [En ligne], 313 | 2020, mis en ligne le 24 septembre 2021, consulté le 25 septembre 2021. URL : http://journals.openedition.org/ edl/3246 ; DOI : https://doi.org/10.4000/edl.3246 


\section{$\grave{A}$ LA RACINE... OU L'HISTOIRE SOUTERRAINE \\ DE LA VENUE DU THÉÂTRE DU SOLEIL À LAUSANNE. PORTRAITS CROISÉS D'OMAR PORRAS ET D'ARIANE MNOUCHKINE}

Spécialiste en études théâtrales, l'auteure de cette contribution est une collaboratrice régulière du Théâtre Kléber-Méleau (TKM) dont elle a écrit tous les programmes des spectacles. Elle a suivi jour après jour l'aventure de l'invitation à Lausanne d'Ariane Mnouchkine par Omar Porras qu'elle retrace ici, intégrant à une parole d'historienne extra-diégétique rassemblant les fils de son enquête les témoignages intra-diégétiques de ce dernier, en une parole à la poétique hybride.

Si nous sommes tous des êtres composites, faits de nos héritages, de nos traversées, de nos expériences, le metteur en scène, comme le chorégraphe, l'acteur ou le danseur imprime peut-être plus fortement encore en lui ce qu'il a reçu dans sa formation, ses rencontres artistiques, intellectuelles et amoureuses, et par des creux ou des pleins, avec des rejets et des ruptures, des attractions et des fusions ou reprises, chacune de ses créations est un résultat alchimique, la radiographie d'une ouvre du répertoire ou d'une thématique, mais aussi les échos mêlés de sa vie.

L'essence de l'artiste ne résiderait-elle pas en cela? Non pas tant dans le surgissement que dans le resurgissement et l'hybridation, ce qui le placerait d'une création à l'autre en situation de restitution de ses innutritions, de ses états d'âme, de fragments de vie, de discussions, mais un resurgissement qui deviendrait très vite rêverie collective, en amont du travail au plateau, comme tout au long du processus de création, dans le frottement des idées de scénographes et dramaturges, créateurs sons et créateurs lumières, interprètes, mais aussi techniciens et autres membres 
du cénacle par lequel se dessine une création dans le champ du spectacle vivant - tout cela dans un vaste jeu de raisons et de hasards ou «accidents".

Lors d'une rencontre au Théâtre La Grange de Dorigny, Omar Porras, à l'origine de la venue du Théâtre du Soleil à Lausanne à l'automne 2018, a éclairé les rhizomes profonds de cette invitation lors d'une séance de séminaire dont je vous propose de suivre plus avant les méandres ${ }^{1}$.

\section{Du pays de Pachamama a au Théâtre du Soleil}

Si la pandémie de la COVID-19 n'avait pas si violemment sévi, la compagnie de cet artiste, le Teatro Malandro, aurait fêté cette année ses trente ans: 2020 semble comme passé à la trappe d'un temps dont le pouls s'est arrêté. C'est en 1990 qu'Omar Porras a choisi de poser ses valises et de construire son aventure théâtrale en Suisse: c'était alors un homme jeune de vingt-sept ans qui, après un détour par Paris, arrivait de Colombie, "terre de chamans, terre d'Indiens, terre des mohanes, des caciques, des zipas et des zaques, qui couvraient leur corps de poudre d'or " ${ }^{3}$, terre façonnée par les mythes, aux "arbres gigantesques" et aux "plantes magiques" ${ }^{4}$. Mais ce même pays a un envers de médaille aussi sombre que l'avers est coloré et fabuleux, sur fond de guerre, de narcotrafic, d'exactions, de corruptions et d'impunités 5 . Difficile, dans cet autre contexte, d'imaginer consacrer sa vie au théâtre? Oui et non, car «les rêves sont le cœur sacré de notre imagination " ${ }^{6}$, nous dit le Poète...

De fait, à dix-neuf ans, après avoir passé son baccalauréat et appris la discipline du soldat, Omar Porras s'est présenté au concours d'entrée de

I. Certaines citations viennent de cet entretien réalisé par les étudiants, enregistré et transcrit par Nino Fournier, doctorant à l'UNIL.

2. Déesse-mère de la fertilité dans la cosmogonie andine, présente dans l'imaginaire de tous en Colombie.

3. F. Melquiot, $M a$ Colombine, p. 13.

4. Ibid. C'est ce que le personnage auto-fictionnel de $\mathrm{Ma}$ Colombine nous explique sous les traits d'un professeur peu amène, au commencement de ce remarquable seul en scène créé le 18 janvier 2019 au Théâtre Am Stram Gram à Genève, et repris un mois durant au Festival d'Avignon de la même année au Théâtre Gilgamesh (le 11 Avignon).

5. Cf. J. J. Lozano et M. Hollman, Impunity.

6. O. Porras, édito du Programme de saison 2016-2017 du TKM. 
l'Escuela distrital de Teatro (École de Théâtre du District de Bogota): il n'y fut pas pris (les dieux nous offrent parfois des chemins de traverse), mais entra à l'Escuela de danza moderna (École de danse moderne) de Bogota, où il découvrit Vaslav Nijinski et Isadora Duncan à travers leurs pas de danse et leur mémoire, tout en étant coursier pour une agence de voyage, une ouverture vers l'envol.

Son rêve de théâtre avait déjà la couleur des étoiles, mais il n'était pas aisé d'y accéder depuis la terre de ses ancêtres. Dans les années 1980, les formations se limitaient à l'École du Théâtre du District de Bogota et à l'École nationale d'art dramatique; le théâtre se pratiquait directement dans des troupes indépendantes créées dix à vingt ans plus tôt comme El Teatro La Candelaria, El TPB (Teatro Popular de Bogota), El Teatro Experimental La Mama, El Teatro El Local ${ }^{7}$, El Teatro Taller de Colombia et El Teatro Libre... ${ }^{8}$ Ce mouvement de théâtre contemporain, appelé Nuevo Teatro, mettait au cœur de ses projets la dimension collective de la création, et ce comme en Europe où au même moment fleurissait Le Théâtre du Soleil, parallèlement aux troupes d'Eugenio Barba, de Peter Brook, de Jerzy Grotowski et du Théâtre de l'Aquarium autour de Jacques Nichet et d'autres élèves de l'École Normale Supérieure de la rue d'Ulm, mais aussi comme aux États-Unis avec The Living Theater, The Bread and Puppet Theater, El Teatro Campesino, la MaMa à New York, et comme au Japon avec Shûji Terayama, Makoto Satô, Jûrô Kara ou encore Shôgo Ôta. Dans ce contexte, l'influence de Bertolt Brecht n'était pas moindre et pour certains, l'enquête de terrain pour collecter la parole du peuple était aussi essentielle. Au-delà de Bogota, d'autres échos encore à cette modernité se faisaient entendre en Colombie avec $E l$ Teatro Experimental de Cali et Enrique Buenaventura9 (une compagnie créée dès 1955) - ce même TEC qui fut invité au Théâtre des Nations

7. Miquel Torres a transmis en 2007 El Local à Omar Porras qui y a implanté un nouveau Théâtre, El Teatro La Quinta Porra, actuellement dirigé par Fabiana Medina.

8. El Teatro La Candelaria a été fondé en 1966 par Santiago Garcia (né en 1928, il s'est éteint le 23 mars 2020 à l'âge de 92 ans), El TPB en 1967 par Jorge Ali Triana (né en 1942), El Teatro Experimental La Mama en 1969 par Eddy Armando (1942-2011), El Teatro El Local en 1970 par Miquel Torres (né lui aussi en 1942), El Teatro Taller de Colombia et El Teatro Libre, créé respectivement par Mario Matallana et Jorge Vargas en 1972 et par Ricardo Camacho, Jorge Plata, Patricia Jaramillo, Germán Moure et Héctor Bayona en 1973.

9. $1925-2003$. 
en 1960-1961, mais qui fut également programmé (comme El Teatro la Candelaria) au Festival de Nancy, notamment en 1971 - deux lieux où le plateau est appréhendé dans sa dimension internationale (en rassemblant des artistes venus des cinq continents) ${ }^{10}$.

Encore tout jeune, Omar Porras a dû sentir ce souffle de l'ailleurs, car il souhaita très vite quitter le pays. Comme pour le personnage de l'auto-fiction $\mathrm{Ma}$ Colombine, Oumar-Tutak-Hijo-de-Chibcha-Vuelode-Condor-Suvan-y-Ven, il «a volé, très vite, volé, très vite » ${ }^{11}$ jusqu'à gagner Paris.

À l'Université de Lausanne, Omar Porras a raconté comment son parcours, ses voyages comme ses recherches, sont «le résultat de rencontres, directes ou indirectes, avec des maîtres qui sont devenus [s]es guides, tels Ariane Mnouchkine, Giorgio Strehler, Jerzy Grotowski, Peter Brook, Eugenio Barba et I Made Jimat» ${ }^{12}$, pour une traversée de sensibilités transnationales d'une Franco-russe à un Indonésien, en passant par un Italien, un Polonais et un Anglais.

Pour ce metteur en scène, toutes ces figures tutélaires l'ont "orienté vers une source inépuisable de connaissances et d'aventures, vers des cultures, des pays lointains qui ont nourri [s]a curiosité d'homme et d'artiste» ${ }^{13}$ :

À peine arrivé de Bogota à Paris en 1984, j’ai eu la chance de découvrir l'influence du théâtre oriental en Europe, à la Cartoucherie, avec le théâtre d'Ariane Mnouchkine et L'Histoire terrible mais inachevée de Norodom Sihanouk, roi du Cambodge avec ces personnages masqués fascinants comme des Shite du Théâtre Nô, interprétés par Georges Bigot et Guy Freixe... ${ }^{14}$

Io. Rien d'étonnant à ce qu'une fois directeur d'une institution vingt-cinq ans plus tard, Omar Porras mette en place un cycle indien aux couleurs du Kathakali, de ses mythes et de ses sonorités, puis travaille à l'invitation du Théâtre du Soleil pour une fête de l'imagination au croisement des cultures.

II. F. Melquiot, $M a$ Colombine, p. 21.

I2. Rencontre retranscrite citée.

13. Ibid.

I4. Omar Porras, propos mis en ligne sur le site du TKM pour l'accueil du Théâtre du Soleil, octobre 2018. 


\section{"Le théâtre a des portes et des fenêtres. Il dit le monde entier» ${ }^{15}$}

L'aventure théâtrale d'Ariane Mnouchkine prend son envol en 1964, alors qu'elle n'a que vingt-cinq ans et qu'elle fonde le Théâtre du Soleil avec ses compagnons de l'ATEP (Association théâtrale des étudiants de Paris) - une exception dans le monde théâtral francophone, le groupe constituant un collectif rassemblant comédiens, artisans, musiciens et personnel administratif qui partagent systématiquement tout travail (artistique, technique, administratif et domestique) et dont les salaires sont tous égaux - un principe maintenu depuis cinquante-quatre ans...

En 1970, Giorgio Strehler met toute sa confiance en ces jeunes artistes à qui il ouvre le Piccolo Teatro de Milan pour la création de 1789; et c'est cette même année que le Théâtre du Soleil investit la Cartoucherie (un ancien atelier des poudres de la Ville de Paris situé dans le Bois de Vincennes) pour y construire un théâtre engagé et populaire, dans l'héritage de la pensée de Jean Vilar. Cette même Cartoucherie est devenue un haut lieu de création regroupant quatre théâtres: le Théâtre du Soleil, le Théâtre de l'Épée de Bois, le Théâtre de l'Aquarium, le Théâtre de la Tempête, ainsi que le CNDC Atelier de Paris - Carolyn Carlson, dédié à la danse contemporaine, et la maison d'ARTA (Association de recherche des traditions de l'acteur).

Investi dans le spectacle vivant, tout comme dans la société qui l'entoure, le Théâtre du Soleil s'est constitué en un modèle comme troupe et comme aventure humaine. Laboratoire, il est en effet devenu une scène unique en son genre, reconnue dans le monde entier pour la force de ses créations (loin de tout réalisme, développant constamment une poésie aux images fulgurantes), une scène ouverte à l'international, souvent nourrie aux sources de l'Orient, toujours en prise avec les préoccupations sociales et politiques de son époque.

Le Théâtre du Soleil est un espace de sacerdoce et de renouvellement constant, la transmission étant au cœur de ses préoccupations; un espace de résistance, fait d'une troupe aujourd'hui de quatre générations, de quatre-vingts à cent personnes, chacun de ses membres (entre autres Afghans, Algériens, Argentins, Arméniens, Belges, Brésiliens, Cambodgiens, Chiliens, Chinois, Espagnols, Français, Hongrois, Indiens, Irakiens, Iraniens, Italiens, Japonais, Russes, Togolais) étant

I5. Ariane Mnouchkine, septembre 2018, au TKM. 
porteur d'un monde, de cultures et traditions particulières, susceptibles d'être transmises au sein du groupe et pouvant alimenter ses créations une diversité porteuse d'universalité.

Le Théâtre du Soleil est un monument du théâtre contemporain : il représente un bastion du théâtre populaire en acte, "élitaire pour tous" comme le disait Antoine Vitez et que se plaît à réaffirmer après lui Ariane Mnouchkine, c'est-à-dire transgénérationnel, transsocial et international, et où le public (fidèle parfois depuis des décennies) est au cour du processus de création, destinataire et partenaire direct tout à la fois.

Citons, après Les Petits Bourgeois (1964), Le Capitaine Fracasse (1966), La Cuisine (1967), Le Songe d'une nuit d'été (1968) et Les Clowns (1969), ou encore 1789, La Révolution doit s'arrêter à la perfection du bonheur (1970), 1793 (1972), L'Âge d'or (1975) et Mephisto (1979), L'Histoire terrible mais inachevée de Norodom Sihanouk, roi du Cambodge d'Hélène Cixous (1985), L'Indiade ou L'Inde de leurs rêves d'Hélène Cixous (1987), un hommage à Gandhi, Les Atrides (1990-1992), avec Iphigénie à Aulis, Agamemnon, Les Choéphores et Les Euménides, nourries de formes indiennes traditionnelles comme le Kathakali et le Kutiyattam, La Ville parjure ou le réveil des Erinyes d'Hélène Cixous (1994), Le Tartuffe (1995) «recontextualisé» dans une Algérie soumise par les islamistes, Et soudain des nuits d'éveil (1997), Tambours sur la digue (1999) d'Hélène Cixous qui se nourrit du Bunraku, l'art des marionnettes du Japon, Le Dernier Caravansérail (Odyssées) (2003) qui met sur scène notre époque de déplacés politiques, de trafics et d'exactions, d'exodes contraintes, en un jeu de miroir saisissant, Les Éphémères (2006), une épopée contemporaine dans l'intime de nos vies sous la forme d'un récit à trente voix (où l'on nous parle par touches entrecroisées de la violence dans les foyers, de l'illettrisme, de la vieillesse et de la mort...), Les Naufragés du Fol Espoir (2010) qui met en scène un tournage dans le grenier d'une guinguette, trois jours avant la Première Guerre mondiale, ou encore Macbeth (2014) qui reprend le fil du cycle fondateur des Shakespeare, de 1981 à 1984 (Richard II, La Nuit des rois et Henri IV).

Parallèlement sont aussi à mentionner une série de films d'importance, réalisés par Ariane Mnouchkine en lien direct avec ses créations scéniques: 1789 (1974), La Nuit miraculeuse (1989), Tambours sur la digue (2002), Le Dernier Caravansérail (Odyssées) (2006), Les Naufragés du Fol Espoir (2013) et bien sûr une incontournable histoire de la vie de celui qui fut plus qu'un homme, un symbole: Molière (1978). 
D'un spectacle ou d'un film à l'autre ${ }^{16}$, le jeu de l'acteur est toujours organique et épique, stylisé, né de l'improvisation et des appuis de la musique de Jean-Jacques Lemêtre réalisée à partir d'instruments du monde entier, mais aussi de son invention. Et toujours le Théâtre du Soleil nous parle de notre époque, de la résistance des peuples, de la force du plateau à représenter le monde d'aujourd'hui, de sa capacité à traverser des questionnements à la fois politiques et humains, des Odyssées toujours au croisement des cultures: Une chambre en Inde, créée à la Cartoucherie en novembre 2016, ne fait pas exception, ni Kanata - Épisode I - La Controverse, mis en scène par Robert Lepage avec la troupe du Théâtre du Soleil, qui fut présenté du 15 décembre 2018 au 17 février 2019 à la Cartoucherie, avec le Festival d'Automne à Paris.

\section{Le Théâtre du Soleil: lieu de révélations}

La découverte par Omar Porras d'un premier spectacle de cette troupe a eu lieu dès 1985 au moment de la création de Norodom Sihanouk: comme il en témoigne volontiers, ce fut pour lui "une révélation". C'était comme rencontrer Pachamama, "comme approcher du Soleil sans se brûler la peau "; une artiste qui "portait en elle tous les théâtres du monde». Par elle, il comprend que sa maison serait le théâtre, sa «maison sans murs ni toit, ouverte aux nuages qui passent, aux géantes, aux anges et aux aigles", cet endroit où «la mémoire et l'imagination, le dedans et le dehors, se rejoignent pour se parler. [...] Le visible et l'invisible. [...] Le silence et la musique. [...] La vérité, le mensonge et les mille nuances entre les deux. [...] Les morts et les vivants. [...]». Le théâtre allait ainsi devenir pour Omar Porras son «microscope» et son "télescope ${ }^{17}$.

Lors de la rencontre avec les étudiants à l'Université de Lausanne d'octobre 2018, le metteur en scène se souvient:

Quand j'ai rencontré Ariane, je venais d'arriver de la Colombie. J'ai quitté mon pays, parce que je n'ai pas pu faire du théâtre

I6. Béatrice Picon-Vallin a retracé en novembre 2014 l'aventure de la troupe dans un ouvrage essentiel intitulé Le Théâtre du Soleil, les cinquante premières années, édité chez Actes-Sud.

I7. F. Melquiot, $M a$ Colombine, p. 28 sq. 
en Colombie: quand j'ai tenté d'en faire, la Colombie avait déjà connu plus de trente ans de guerre civile, et tout ce qui ressemblait à l'opposition, tout ce qui contrariait ce pseudo-système démocratique latinoaméricain était menacé...

Faire du théâtre était un symbole d'opposition à l'État, et les groupes de théâtre engagés, les théâtres qui rassemblaient un grand public, un public populaire, étaient menacés: l'art était pris en otage par un État très élitiste et corrompu. Il y a eu des morts et des tortures; il y a eu des menaces de mort et des exils.

Je n'ai pas pu faire du théâtre en Colombie: j'ai dû quitter le pays pour en faire pour ces raisons, mais ce qui me dérangeait aussi là-bas, c'est que pour faire du théâtre, il fallait avoir un drapeau dans la main. Que ce soit un drapeau vert, rouge ou jaune - peu importe. J'avais besoin de mes deux mains libres pour voler.

En rencontrant le Théâtre du Soleil et en voyant pour premier spectacle de cette troupe l'Histoire terrible, mais inachevée de Norodom Sihanouk, roi du Cambodge, le jeune homme se rendit cependant compte que le théâtre pouvait être politique sans drapeau. Il entendait au Théâtre du Soleil le même discours que dans El Teatro La Candelaria de Santiago Garcia... Ce qu'Ariane Mnouchkine faisait, c'était bien cela, un acte politique.

Les deux années qui suivirent, de 1985 à 1987, le jeune comédien colombien travailla à intervalles réguliers au Théâtre de l'Épée de Bois - dans Caligula de Camus, puis dans Volpone ou le Renard de Ben Jonson, deux mises en scène d'Antonio Diaz-Florian, mais aussi auprès de Ryszard Cieslak pour un spectacle sur Dostö̈evski : Mon pauvre Fedia. C'est dans cette période qu'il commença le travail du jeu masqué, précisément, parce qu'avec Ariane Mnouchkine, nous retrouvons la filiation, comme dirait Guy Freixe, avec Jacques Lecoq (et Jacques Copeau) ${ }^{18}$, et qu'il savait que «les stages et toutes les créations pratiquement commencent à partir du jeu masqué» - «elle avait fait venir de grands maîtres balinais".

Vint le stage qu'il fit en 1987 avec Ariane Mnouchkine (auquel participait aussi Duccio Bellugi, le plus ancien acteur aujourd'hui du Théâtre du Soleil). Omar Porras fut fasciné de voir comment, «assise sur un petit tabouret, elle orientait un à un ses cinq cents participants, comment elle

I8. G. Freixe, La Filiation Copeau-Lecoq-Mnouchkine. 
fédérait son groupe; comment elle construisait un univers, extrêmement exigeant ${ }^{19}$.

S'il ne trouva pas sa place comme acteur dans ce contexte de grande rivalité, il demanda l'autorisation de rester pour assister de l'extérieur aux séances, pour la voir travailler, pour l'observer dirigeant cette peuplade qui allait participer à la naissance d'une épopée, ce qui lui fut accordé. Rétrospectivement, pour Omar Porras, ce fut comme «la première graine» de son profond intérêt pour la mise en scène.

Ariane Mnouchkine, un modèle ou "une vision, une apparition, un miracle..."

Parallèlement à sa participation dans des créations au Théâtre de l'Épée de Bois dirigé par Antonio Díaz-Florián ${ }^{20}$ (lui-même né dans les Andes péruviennes), le jeune homme d'alors faisait du théâtre de rue à Paris, et bientôt aussi à Berlin, Zurich, Genève:

J'ai traversé plusieurs continents et rencontré de grands maîtres, mais Ariane est toujours restée un modèle - ce qui m’a aidé à tenir le coup, parce que cela fait quarante ans que je fais du théâtre... Son théâtre est pour moi une vision, une apparition, un miracle... Faire du théâtre est un métier extrêmement exigeant; c'est un métier difficile; c'est un métier où l'on est tout le temps entouré de monde, mais il y a beaucoup de solitude...

C'est aussi en voyant Ariane Mnouchkine que j'ai senti qu'il fallait faire du théâtre en troupe - d'où l'idée de fonder une compagnie, quand j'ai décidé de devenir metteur en scène. Je ne voulais pas faire du théâtre en appelant ponctuellement des acteurs: mon rêve était de faire une troupe ${ }^{21}$.

\section{I9. Ibid.}

20. En 1969, Antonio Diaz-Florian reprend le théâtre de l'Épée de Bois dirigé par Tania Balachova qui était situé à l'époque à Paris, rue de l'Épée-de-Bois dans le $5^{\mathrm{e}}$ arrondissement, au coin de la rue Mouffetard. Il y fonde l'atelier de l'Épée de Bois, un groupe de théâtre expérimental, qui devient plus tard le Théâtre de l'Épée de Bois de la Cartoucherie.

2I. Rencontre retranscrite citée. 
C'est ainsi qu'Omar Porras a développé un langage qu'il transmet volontiers dans des stages - notamment à ARTA et aux Ateliers Carolyn Carlson (à Vincennes), à l'ESAD (École supérieure d'Art dramatique, à Paris), à l'École Serge Martin (à Genève), aux Teintureries et à la Manufacture (à Lausanne) -, mais qui fonctionne avant tout d'une création à l'autre comme un langage commun à partager. La première phase du travail consiste en un training qui vise à la prise de conscience de "l'équilibre» qui va contribuer à appréhender un état particulier, celui de "la présence», mais aussi la gestion du corps dans l'espace et la mise en place d'une dynamique qui permettront d'harmoniser l'ensemble du travail au plateau. Un deuxième temps se nourrit d'improvisations et de l'organisation d'un langage singulier de chaque acteur, le vecteur pour esquisser les contours de son personnage. «C'est le corps qui devient pinceau, qui dessine sur la page blanche de la scène, et fait surgir l'idéogramme", dit-il volontiers: le "corps extra-ordinaire, extra-quotidien"; le geste qui se fait poème ou encore "méditation en mouvement». La troisième phase de la recherche vise à confronter les alphabets gestuels, le langage poétique de chacun, en vue de les faire se rencontrer: dans le collectif, le singulier de l'acteur-poète se fait monde. Ces phases peuvent se moduler. Elles peuvent commencer par un training plutôt inspiré des marches de Tadashi Suzuki ou du Kalaripayatt; elles peuvent s'épaissir par un travail de jeu masqué: toujours elles aident à consolider une troupe, même si de configuration mouvante... L'idéal de la troupe a été un fil rouge pour Omar Porras, dont la constitution, évolutive, a pu se faire à travers cette méthode structurée et empirique tout à la fois du plateau, d'une création à l'autre, trente ans durant, en Suisse, en France et dans d'autres pays d'Europe, mais aussi en Colombie et au Japon, au Mexique et au Venezuela.

En revenant sur le parcours de l'artiste, l'influence considérable d'Ariane Mnouchkine dans son imaginaire devient saillante: depuis les années 1980, avec une profonde admiration, Omar Porras a continué à suivre de près "la grande sagesse et le profond engagement que cette artiste a su cultiver à la Cartoucherie de Vincennes » ${ }^{22}$, où des milliers de spectateurs viennent de tous les coins du monde découvrir des créations qui ont marqué plusieurs générations. 


\section{La genèse du projet: le 3 décembre 2016, du spectacle à l'invitation}

L'idée d'accueillir Le Théâtre du Soleil à Lausanne a germé comme une évidence le 3 décembre 2016, lorsqu'Omar Porras a assisté à une représentation d'Une chambre en Inde à La Cartoucherie (un spectacle qui venait d'être créé le 5 novembre 2016). En nous invitant dans une chambre, en Inde, au cœur battant de l'humanité, Ariane Mnouchkine et sa troupe partageaient avec nous dans ce spectacle ce désarroi qui pouvait être le nôtre face au chaos du monde, pour mieux cicatriser nos peurs par un rire salvateur et réaffirmer l'importance de l'art, la force des messages de paix et d'amour face au pire.

Une chambre en Inde, c'est l'histoire de Cornélia qui doit prendre au pied levé la tête de sa troupe de théâtre et y remplacer le metteur en scène, Constantin Lear, sous le choc des attentats de Paris de novembre 2015. Or le sujet du spectacle à créer, encore non déterminé, est à annoncer dès le lendemain. Quel pourrait-il être? "Le sort abominable fait aux femmes"? "La pauvreté"? "La montée des violences"? "La guerre de l'eau»? "Les victoires des théocraties"? "La faiblesse des démocraties qui ne défendent plus leurs valeurs"? Comment raconter «le chaos d'un monde devenu incompréhensible »? À chaque songe ou rêverie performative, un nouveau spectacle s'esquisse... Et en même temps, au milieu de ce maelström de sujets névralgiques, traversent ce plateau atemporel aussi bien Anton Tchekhov accompagné des trois sœurs Irina, Macha et Olga, que Shakespeare, Gandhi, ou encore Chaplin et son Dictateur, l'humour n'étant jamais très loin pour transcender nos peurs et nos colères: le théâtre porte en lui une force régénératrice, une puissance de vie inépuisable...

Pour aborder les désordres du monde, il s'est agi de travailler à partir d'improvisations et, ce faisant, de dessiner une partition collective imprégnée de ce qui hantait alors notre actualité - de Daech aux questions écologiques - et fondée sur les propositions de chacun et de chacune. C'est collectivement que sont nées les propositions dramaturgiques en harmonie avec Hélène Cixous, comme celles liées à la technique, orchestrées par Ariane Mnouchkine, quand la musique du spectacle était à la fois l'œuvre de Jean-Jacques Lemêtre et issue des traditions musicales du Tamil Nadu.

Pour autant - alors que les premières répétitions ont eu lieu en Inde, à Pondichéry, au Théâtre Indianostrum de Koumarane Valavane - 
est donnée à voir au cœur du spectacle la grande épopée indienne du Mahabharata à travers les épisodes du viol de Draupadi et de la mort de Karna en Terukkuttu (une forme théâtrale populaire ancestrale et toujours vivante, immuable et d'une beauté inouie) - où évoluent les personnages de Draupadi, du roi Duryodhana, de Dushasana, des cinq Frères Pandavas, de Karna, de Ponnourouvi et même du dieu Krishna - sur fond d'une rythmique née du chant carnatique, des percussions thalam, mridangam et dolak, de l'harmonium et du mukhaveenai (hautbois tamoul). Kalaimamani Purisai Kannappa Sambandan Thambiran, maître et dépositaire de l'art du Terukkuttu, a été en effet partie prenante de cette grande aventure, assisté de Palani Murugan.

Le principe d'enchaînement des tableaux de ce spectacle épique pourrait s'assimiler au fondu enchaîné cinématographique: les spectateurs sont face à une chambre d'écho du monde en un kaléidoscope de visions cathartiques, où trente-quatre comédiens, de treize nationalités différentes, nous saisissent, nous enthousiasment par la vitalité et la précision de leur jeu, puisent au plus profond de notre humanité et font fleurir, derrière le cauchemar, le rêve, réactivent à travers l'art notre croyance inébranlable en la beauté et en l'humanité.

Au sortir de cette représentation du 3 décembre 2016, Omar Porras s'est entretenu avec Ariane Mnouchkine qui lui a alors confié combien elle aurait plaisir à venir jouer en Suisse, avec sa complicité:

Je sors du spectacle, j'étais en larmes devant elle. Je lui prends les bras, lui dis ce que j'ai senti du spectacle, puis ajoute: "Ariane, est-ce que tu m'autorises à aller à Lausanne et à dire à mes collègues que ce spectacle, il faut qu'il soit vu en Suisse?» Et elle me répond: «Oui, Omar. J'aime Lausanne...» Elle appelle son directeur technique, Étienne Lemasson, et lui dit: «Donne à Omar les plans de tournée». Étienne me les donne... Cette nuit-là, je n'ai pas dormi ${ }^{23}$.

C'est ainsi qu'est né ce projet ambitieux de programmer Une chambre en Inde dans la région lausannoise, à la condition qu'un lieu adéquat soit trouvé pour permettre cet accueil. L'idée de faire venir le Soleil à Lausanne comblait un désir de partager sur ce territoire, la Suisse, qui est devenue son pays, un travail théâtral d'exception: Omar Porras était en Suisse depuis près de trente ans, et depuis un peu plus d'une année

23. Ibid. 
(depuis septembre 2015), directeur du TKM-Théâtre Kléber-Méleau, à Renens - avec à ses côtés une administratrice hors pair, Florence Crettol, qui comme Étienne Lemasson au Théâtre du Soleil, est le «Grand intendant» de ses entreprises. Pour lui, faire venir Ariane Mnouchkine, c'était comme s'il s'y était préparé depuis toujours: «il a fallu presque vingtcinq ans...». Quelques semaines plus tard, en février 2017, le syndic de Lausanne, les représentants de la culture de la Ville de Lausanne, du Canton de Vaud et de la Ville de Renens, ainsi que la Présidente du Conseil de Fondation du TKM, ont fait le déplacement à Paris pour découvrir Une chambre en Inde sur son lieu de création, La Cartoucherie. Tout ceci était comme un conte, tant et si bien que sur le chemin, toute cette délégation manqua de se perdre dans la forêt, ou plutôt le Bois de Vincennes - un épisode cocasse -, avant de pouvoir assister, in extremis, à une représentation, d'en sortir fascinée et d'être absolument conquise par le projet de cet accueil.

Le Théâtre du Soleil était venu en Suisse romande en 1970 avec son spectacle 1789, La Révolution doit s'arrêter à la perfection du bonheur, puis en Suisse alémanique, à Bâle, en 2000, avec Tambours sur la Digue. Revenir en Suisse romande avec Une chambre en Inde représentait un événement historique pour le territoire en général et pour Lausanne en particulier. Chaque soir, six cents spectateurs allaient avoir le plaisir de cette traversée bouleversante, à un tarif qui honorerait l'esprit de Jean Vilar, une condition à laquelle tenait mordicus Omar Porras. Le budget d'accueil alors prévu l'était pour soixante-cinq personnes en tournée à transporter, loger et défrayer, avec un prix de cession considérable par représentation. À cela, il fallait ajouter les frais liés à la location de l'espace, à la décoration de l'entrée, à l'installation du bar et du restaurant, au montage du décor et à l'exploitation du lieu pendant un mois (personnel d'accueil, sécurité, etc.). Ce budget prévoyait un prix de la place au tarif plein de CHF 50.- décliné pour les différentes réductions (jeunes, AVS, etc.), soit un prix moyen du billet de CHF 27.- et un taux global de fréquentation de $85 \%$ - qui en réalité a atteint les $100 \%$ !

Pour rendre possible la réalisation de ce projet, il fallait s'organiser très sérieusement. C'est ainsi que fut créée "L'Association pour la venue d'Une chambre en Inde", fédérant, sous l'impulsion du TKM et de son directeur, les autorités publiques de Lausanne, de Renens, du Canton de Vaud et de plusieurs théâtres romands: le Théâtre de Carouge-Atelier de Genève, la Comédie de Genève, le Théâtre Benno Besson d'Yverdon- 
les-Bains, le Théâtre du Passage de Neuchâtel, le Théâtre Forum Meyrin, le Théâtre populaire romand de la Chaux-de-Fonds, le Théâtre VidyLausanne et l'UNIL-La Grange de Dorigny. Tous ont contribué à hauteur d'au moins 10'000 CHF et ont acheté des billets en prévente - le spectacle était inscrit dans leur propre programmation. Ensemble - ce fut le maître mot de cette aventure -, tous ces partenaires sont parvenus à rassembler les fonds publics et privés nécessaires (deux millions deux cent mille francs suisses) pour rendre possible la mise en place d'un lieu avec une scène, des loges à vues, un espace de restauration comme à la Cartoucherie lors de ces vingt-et-une représentations, devant 12'600 spectateurs. Comme le disait Ariane Mnouchkine lors de la conférence de presse du 17 avril 2018 organisée au TKM:

Quand on nomme tous ces théâtres, tous ces amis, que nous ne connaissons pas pour certains, qui se sont fédérés, qui se sont unis, pour faire venir un théâtre comme le nôtre, c'est extrêmement émouvant. Je pense que c'est important, également pour d'autres théâtres, que c'est important pour le Théâtre, parce que cela veut dire que c'est possible, qu'il y a eu un désir ardent, qu'il y a eu du travail et il ne peut pas y avoir de travail sans désir. C'est-à-dire qu'il y a eu au fond une véritable politique culturelle, menée par des institutions, par des hommes et des femmes politiques. Il y a eu une conjoncture, une conjonction, une coalition, mais peut-être même une conjuration du public et du privé pour faire venir quelque chose de "minuscule», une troupe de théâtre... Nous sommes «minuscules», mais voilà, un maire est venu chez nous, des directeurs, des directrices, et ont décidé que ce «minuscule» est important; qu'il est vital.

Le Théâtre du Soleil bénéficie d'un grand plateau, avec des caractéristiques particulières, et sa salle peut accueillir une jauge de 560 spectateurs. À la Cartoucherie, dès l'entrée au Théâtre, le public est immédiatement plongé dans l'univers de la pièce: l'Inde. Il est accueilli chaleureusement par un repas et des spécialités indiennes. Une librairie propose divers ouvrages sur le Théâtre du Soleil et des questions saillantes pour aborder notre monde. Les loges sont à vue, sous le gradin, et le spectateur peut entrer dans l'intimité des comédiens qui se préparent pour leurs rôles. Tous ces éléments sont reconstitués en tournée. Il fallait donc trouver un lieu idéal et suffisamment vaste pour pouvoir restituer l'univers de La Cartoucherie. 
Plusieurs possibilités ont été envisagées: la transformation du plateau du TKM, la location de la nouvelle patinoire provisoire de Malley ou de la Halle 10 de Beaulieu. Finalement, la plus grande Halle du Centre des Congrès - Palais de Beaulieu, la Halle 7, a été retenue et validée par Ariane Mnouchkine et son équipe après une visite sur place, le 17 mai 2017. Ce lieu, très accessible en transport en commun ou en voiture (avec son grand parking), bénéficie de toutes les commodités avec un accès pour les personnes à mobilité réduite entre la salle, le bar, les toilettes et les vestiaires, et la circulation du public se fait facilement: Tous ces aspects ont été considérés pour valider ce choix, jusqu’à la température de la chaufferie pour éviter que le dernier rang ne souffre de la chaleur! Ce sont ensuite des dizaines d'hommes et de femmes qui ont reconstitué la Cartoucherie de Vincennes, qui ont construit le même type de gradin avec le même angle, la même profondeur et la même hauteur, dès septembre 2018. Ariane Mnouchkine savait exactement ce qu'elle souhaitait: elle a été engagée dans le montage, avec une exigence exemplaire, au centimètre près. Puis, durant un mois entier et pour vingt-et-une représentations (cinq par semaine), du 24 octobre 2018 au 18 novembre 2018, la Suisse a accueilli cette troupe emblématique de l'histoire du théâtre demandée dans le monde entier et qui n'accepte qu'exceptionnellement des lieux de tournée, et c'était la première fois que se sont rassemblés autant de théâtres autour d'un projet qui était devenu plus qu'un important projet fédérateur, une fête humaniste, car, pour reprendre les mots d'Omar Porras:

Une chambre en Inde est à la fois un océan d'espoir et un tonnerre de lumière qui nous invite à ouvrir les yeux de notre conscience et à éveiller notre humanité ${ }^{24}$.

Deux ans plus tard, toutes ces visions nous accompagnent toujours, et Ariane Mnouchkine est devenue aussi pour nous tous, ses spectateurs, un modèle, pour son sens du sacré, sa rigueur, son choix politique de la troupe, sa façon de croiser imaginaire et réel au plateau et, en nous rendant plus forts, de nous inviter, in fine, au voyage de nos vies.

Brigitte Prost Université Rennes 2 et Théâtre Kléber-Méleau

24. Ibid. 


\section{BIBLIOGRAPHIE}

Freixe, Guy, La Filiation Copeau-Lecoq-Mnouchkine, Montpellier, Éditions de l'Entretemps, 2014.

Melquiot, Fabrice, Ma Colombine, Paris, 2019 (Avant-Scène Théâtre, 1460).

Porras, Omar, édito du Programme de saison 2016-2017 du TKMThéâtre Kléber-Méleau.

VILAR, Jean, "Le TNP, service public», in Le Théatre, service public et autres textes, éd. par Armand Delcampe, Paris, Gallimard, 1986.

\section{Filmographie}

Lozano, Juan José, Hollman, Morris, Impunity, prod. Intermezzo Films, distrib. Nour Films, 2010, 85'. 